\title{
The segmental conic model for forest measurements
}

\author{
Gildardo Cruz de León ${ }^{1}$ y José Cruz de León ${ }^{1}$
}

\begin{abstract}
A close analysis of traditional geometries and volume equations in forest measurements lead us to propose the use of conic geometry by segments as an alternative method to approach the whole form of any regular tree bole, log or bolt. The model is general and applies to any solid of revolution and is supported on the truncated cone volume equation. It is called The Segmental Conic Model. The model accuracy depends on the number of segments and is exact in the limit when that number goes to infinity.
\end{abstract}

KEY WORDS:

Cubic volume, mathematical analysis.

\section{RESUMEN}

Un análisis minucioso de las geometrías tradicionales y ecuaciones de volumen en mediciones forestales nos conduce a proponer el uso de la geometría cónica por segmentos como un método alternativo para aproximar la forma completa de cualquier tronco de árbol en pie, en rollo, o troza. El modelo es general, se aplica a cualquier sólido de revolución y se apoya en la ecuación de volumen de cono truncado. Se denomina el Modelo Cónico Segmentado. La precisión del modelo depende del número de segmentos y es exacta en el límite cuando dicho número tiende a infinito.

PALABRAS CLAVE:

Volumen cúbico, análisis matemático.

\section{INTRODUCTION}

Tree bole volume is a function of its geometry whose accurate determination represents a complex problem. The easiest option is to model it by parts as it is traditionally done in elementary wood and forest literature. Tree boles are modeled geometrically as solids of revolution generated by the function, $y^{2}=A x^{n}$ where $A$ is a constant and $n=0,1$, 2 and 3 . In this way, we have for $n=0$ a cylinder, for $n=1$ a paraboloid, for $n=2$ a cone and, for $n=3$ a neiloid. The literature provides formal calculations of tree bole and log volumes related to each one of those geometries (Castro, 1955; Diéguez-Aranda et al., 2003; Rohman-de la Vega et al., 1994).

In practice, the measurements are obtained through standard empirical methods. The known standard methods are: the Smalian, the Huber and the Newton methods. The most accurate is the Newton method which has the property that it yields, as particular

1 Facultad de Ingeniería en Tecnología de la Madera, Universidad Michoacana de San Nicolás de Hidalgo, Apdo. Postal 580, C. P. 58000, Morelia Mich., México. Telephone: (443) 32235 00Fax: (443) 32679 gcruzl@zeus.umich.mx deleon@zeus.umich.mx 
cases, the corresponding volumes for all the former geometries.

If we call $S$ and $S^{\prime}$ to the crosssectional areas at large and small ends of a log of length $L$, respectively, $S_{1 / 2}$ to the corresponding cross-sectional area at the midpoint of the log, and $V_{N}$ to the volume parameter related to the Newton volume equation (NVE), that equation is derived in the form

$$
\text { [1] } V_{N}=\left[\left(S+4 S_{1 / 2}+S^{\prime}\right) / 6\right] L
$$

which, can also be written as

$$
V_{N}=(2 / 3) V_{H}+(1 / 3) V_{S}
$$

where, $V_{H}=S_{1 / 2} L$ and $V_{S}=\left(S+S^{\prime}\right) L / 2$ are the Huber and Smalian formulas, respectively.

In any method, the volume is computed as an average cross-sectional area times the length. Huber's formula assumes that the average cross-sectional area is found at the midpoint of a log, while Smalian's method assumes that it is given by the average of the two end cross-sectional areas (Avery and Burkhart, 2002). For perfect cylinders and paraboloids, all the three formulas provide identical, perfect results, i.e. $V_{N}=V_{H}=V_{S}$.

\section{OBJECTIVE}

To determine an alternative method for approaching tree bole geometry and volume measurements in forest and wood science.

\section{METHODOLOGY}

The present work is of theoretical nature and represents in itself the development of a method. The results are analytical and have been developed from a detailed analysis of forest and wood measurement literature using elementary mathematical methods such as integral calculus, geometry, and algebra.

The fundamental principle of integral calculus to compute the area below a curve $y=f(x)$ for an interval $[a, b]$ of the variable $x$ is commonly explained as follows. Divide that interval in a number $n$ of segments $\left[x_{i-1}, x_{i}\right]$ of equal length, $\Delta x=(b-a) / n$, where $i=1,2, \ldots, n, x_{0}=a$ and, $x_{n}=b$, and associate to them rectangles of heights $f\left(x_{\mathrm{i}}^{*}\right)$ where $x_{\mathrm{i}}^{*}$ can be any extreme or intermediate point at the segments. The area is approached by the sum of those rectangle areas

$$
\sum_{i=0}^{n} f\left(x_{i}^{*}\right) \Delta x
$$

As the number $n$ increases the approximation is better and in the limit when that number becomes infinity the exact area is obtained. In that limit the sum is defined as an integral and is written as

$$
\int_{a}^{b} f(x) d x
$$

In fact, the segments can have different length and the condition would be that in the limit when their number goes to infinite, the length of the largest one goes to zero (Courant and John, 1965).

A solid of revolution is generated by a rotation of the curve $y=f(x)$ around the $x$ axis. The same rotation of the $n$ rectangles used in the area approximation produces an approximation to the solid of revolution volume by means of $n$ cylinders and the exact volume when $n$ goes to infinity (Stewart, 2002)

\section{RESULTS}

\section{The Segmental Conic Model}

There is a particular way of rectangle selection to apply the approximation 
method previously explained. Relate to each segment a rectangle whose area is the average area of the two rectangles of heights $f\left(x_{\mathrm{i}-1}\right)$ and $f\left(x_{\mathrm{i}}\right)$ corresponding to the extremes of each segment. The result is equivalent to an area approximation by means of trapezoids where a straight line connects the point $\left(x_{\mathrm{i}-1}, f\left(x_{\mathrm{i}-1}\right)\right)$ to $\left(x_{\mathrm{i}}, f\left(x_{\mathrm{i}}\right)\right)$ for each segment and is called the trapezoid's rule (Stewart, 2002).

A $2 \pi$ rotation of the trapezoids results in a solid of revolution volume approximation by means of truncated cones. Any of both options using truncated cones of the same or different length will be called here The Segmental Conic Model (SCM). The model is supported on the truncated cone volume equation (TCVE).

The simple figures for perfect cone and cylinder are particular segment cases of SCM. Then, for any figure resulting from a superposition of truncated cones, cones and cylinders, SCM would be an exact model. Furthermore, we assume that in the most general situation, SCM is exact for any solid of revolution in the limit when the number $n$ of segments goes to infinity. We state and prove formally these assumptions in the following two theorems and a corollary.

\section{Theorem I}

If scm applies exactly to a single truncated cone then SCM applies exactly to a single perfect cone and cylinder.

Proof. By definition, the unitary block of SCM is a truncated cone and its volume is given by TCVE. Then, it is enough to prove that cone and cylinder volumes can be derived from TCVE. If $s$ and $s^{\prime}$ are the cross-sectional areas for the larger and smaller ends of a truncated cone of length $l$, and $d$ and $d^{\prime}$ are the corresponding diameters. It can be formally shown that TCVE is given by the equation
[2]

$$
v=\frac{\pi l}{12} \frac{\left(d^{3}-d^{\prime 3}\right)}{\left(d-d^{\prime}\right)}
$$

That equation can be transformed algebraically to get

$$
v=\frac{\pi l}{12}\left[d^{2}+. d^{\prime 2}+d d^{\prime}\right]
$$

In particular, equations [2] and [3] are well-known in relation to a log in the form of a truncated cone in forest and wood science. If we mathematically apply the limit when $d^{\prime}$ goes to zero to equations [2] and [3], we obtain the volume for a cone. Similarly, applying the limit when $d$ ' goes to $d$ to equation [3] the volume for a cylinder is obtained.

\section{Corollary}

If ScM applies exactly to a single truncated cone, cone and cylinder then SCM applies exactly to any figure resulting from a superposition of truncated cones, cones and cylinders.

Proof. From theorem I, TCVE provides exactly the volumes for a truncated cone, cone, and cylinder. It implies that in any superposition of those simple figures, each one will correspond to a segment in SCM and the total volume will be given exactly by the sum of the individual segment volumes.

\section{Theorem II}

If SCM applies to any solid of revolution geometry then SCM applies exactly to any solid of revolution in the limit when the number of segments goes to infinity.

Proof. It has been stated that SCM can be applied to any solid of revolution as an approximation which is better when 
the number $n$ of segments increases. Then, the total estimated volume is given by the sum of the $n$ individual segment volumes. For the sake of simplicity, suppose segments of equal length. The limit of SCM when $n$ goes to infinity implies the limit when $d$ ' goes to $d$ for any segment what, from theorem I, give us cylinders of infinitesimal length as a limit case and the total volume can be obtained as the limit of the sum of the individual cylinder volumes when $n$ goes to infinite which is exactly the integral's standard definition applied to a solid of revolution. Then, we recover the fundamental principle of integral calculus applied to solids of revolution which provides exact volume determinations.

\section{Application of SCM to a sphere}

For a sphere of unitary radius the exact volume given by integral calculus is.

$$
V=(4 \pi / 3) \approx 4.1888
$$

If the sphere geometry is approached by ten cylinders of length (1/5) and their radii are taken at the middle points of the corresponding segments the resulting volume is 4.2097 (Stewart, 2002). If SCM is applied to that sphere for the same ten segments, using TCVE, the resulting volume is 4.0594 . For the latter case both end segments are cones.

\section{Application of SCM to Forest Measure- ments}

For practical applications to forest measurements, a ScM with a finite number of segments of different length could be well suited to approach the whole tree bole geometry. For instance, figure 1 shows a qualitative approach using only truncated cones and a cone at the top.
Again, the approximation level depends on the number of segments regarded.

It is known that log volumes for the four tree geometries regarded in this work are particular cases of NVE and, inversely, their volume equations can get the form of NVE. In the following theorem an original proof of the latter sentence for the case of a truncated cone is provided.

\section{Theorem III}

If TCVE is known, then TCVE can get the form of NVE.

Proof. The direct form of TCVE obtained from integral calculus is given by equation [2] and it can be transformed to equation [3]. Managing the terms in equation [3] differently we arrive at the equation

$$
v=\frac{\pi l}{12}\left[\left(d+d^{\prime}\right)^{2}-d d^{\prime}\right]
$$

From truncated cone model geometry it is found that the diameter at the log middle, $d_{1 / 2}$, where the crosssectional area is $s_{1 / 2}$, is given by $d_{1 / 2}=\left(d+d^{\prime}\right) / 2$ Using this key fact and rearranging terms in equations [3] and [4], results,

$$
v=\frac{2}{3} v_{S}+\frac{\pi}{12} d d^{\prime} l
$$

and,

$$
v=\frac{4}{3} v_{H}-\frac{\pi}{12} d d^{\prime} l
$$

where $V_{H}=s_{1 / 2} l$, and, $V_{H}=\left(s^{+}+s^{\prime}\right) l / 2$, are the Huber and Smalian formulas for the truncated cone, respectively. Finally, if we add equations [3'] and [4'] and divide by two, we get a form of expressing the TCVE 
in terms only of both the Smalian and Hubber formulas as

$$
v=\frac{2}{3} v_{H}+\frac{1}{3} v_{S}
$$

which has exactly the form of NVE [1'], and completes the proof of the theorem. In this case, NVE has been derived exactly with no assumptions.

\section{CONCLUSIONS}

SCM proposes an alternative method to model physically the whole figure of any solid of revolution and in particular of any regular tree bole, log, or bolt. In principle, the method can be used to approach those geometries by a convenient superposition of truncated cones, cylinders and a cone and its accuracy will depend on the number of segments regarded particularly for the more curved regions. For tree bole segments or log geometries close to cylindrical and truncated cone SCM allows the use of different forms of TCVE to approach volumes in forest measurements only with the knowledge of end diameters and lengths of segments. SCM does not contradict the previous knowledge on the subject and connects to it through the shown theorems. SCM exactly means the application of the trapezoid's rule of calculus to solids of revolution. SCM becomes exact in the limit when the number of segments goes to infinity where it recovers the fundamental principle of integral calculus applied to solids of revolution. In summary, SCM has a solid mathematical support.

In forest measurements there are a lot of more complex geometrical models than those discussed in this work

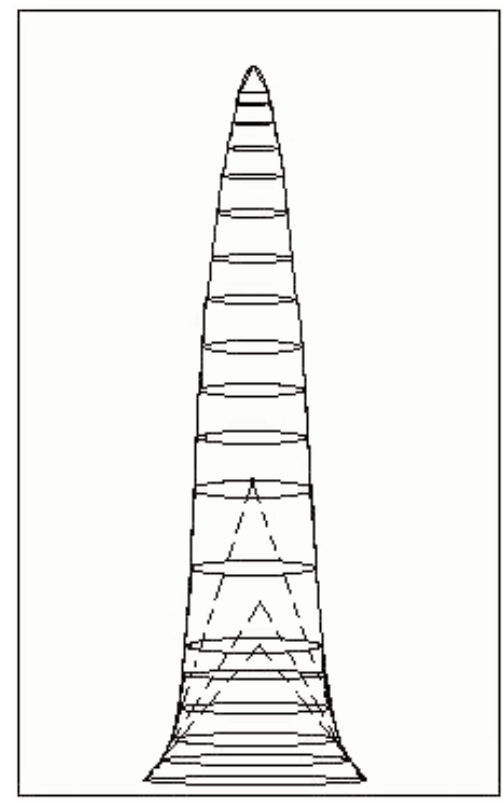

Figure 1. The Segmental Conic Model. The model approaches any particular tree bole geometry by means of segments in the form of truncated cones, cylinders and a cone at the top. In particular, in this figure cylinders were not used. 
(Diéguez-Aranda et al., 2003; West, 2004). Let us remark here that, SCM is a general model in the sense that it can be applied to any solid of revolution. Then, SCM can be applied to any solid of revolution related to forest measurements.

This work has been focused mainly on the formal introduction of SCM, its mathematical foundations, and a qualitative analysis on its application to forest measurements. A further quantitative analysis as function of the number of segments comparing the results to those given by other methods is in progress.

\section{ACKNOWLEDGMENTS}

GCL aknowledges Mexican Board of Education (SEP) for the support of this research through the PROMEP program. Both authors acknowledge Kimberly Brooks for her assistance with the language and to a referee for the suggestion of the reference (Diéguez-Aranda et al., 2003).

\section{REFERENCES}

Avery, T.E. and H.E. Burkhart. 2002. Forest measurements. Mc Graw-Hill, New York.

Castro, F. 1955. Curso de dasometría. ENA, Chapingo, México.

Courant, R. and F. John. 1965. Introduction to calculus and analysis. Volume I. Wiley Internacional Edition, USA.

Diéguez-Aranda, U., M. Barrio-Anta, F. Castedo-Dorado, A.D. RuizGonzález, M.F. Álvarez-Taboada, J.G. Álvarez-González y A. RojoAlboreca. 2003. Dendrometría. Fundación Conde del Valle de Salazar-Ediciones Mundi Prensa, España.

Rohman-de la Vega, C.F., H. RamírezMaldonado y J.L. Treviño-García. 1994. Dendrometría. Universidad Autónoma de Chapingo, México.

Stewart, J. 2002. Cálculo. Trascendentes tempranas. Thomson Learning, México.

West, P.W. 2004. Tree and forest measurement. Springer-Verlag, Berlin. 